\title{
OPTICAL VERSUS CONVENTIONAL IMPRESSIONS OF THE COMPLETELY EDENTULOUS ARCHES
}

\author{
Mohamed Ahmed Alkhodary*
}

\begin{abstract}
Introduction: Digital or optical impressions are becoming of increased use in prosthodontics, however, some studies reported that they were not able to trace mobile and smooth tissues of the edentulous arches. This study compared the optical impressions to the conventional impressions of the edentulous mandibular and maxillary arches via a digital superimposition process.

Materials and Methods: Optical impressions using the Omnicam, and conventional impressions using green compound peripheral molded individual trays and Zinc-Oxide eugenol impression material were made for 18 completely edentulous male patients. The master stone casts were scanned using the Kavo bench top scanner, and then a digital superimposition process of the master casts scans and the optical impressions was carried out using the Geomagic software, which presented the impressions deviations as surface color maps, with each color representing a $0.1 \mathrm{~mm}$ positive or negative deviation. In the maxillary arches 12 readings were taken from the buccal vestibules, residual ridges, median palatine raphes and soft palates, and 24 readings were taken from the hard palates. In the mandibular arches, 12 readings were taken from the buccal vestibules, lingual vestibules, residual ridges, retromolar pads, and buccal shelves of bone. Statistical analysis of the results was done using the Kruskal-Wallis test.
\end{abstract}

Results: In the maxillary arches, significant deviations of the optical impressions from the conventional impressions were found in the buccal vestibules, and in the mandibular arches, significant deviations were found in all the anatomical regions except for the residual ridges.

Conclusion Optical impressions were not able to register the functional depth of sulcus in the maxillary and mandibular edentulous arches, neither the selectively pressed buccal shelves of bone and retromolar pads in the mandibular edentulous arches.

KEYWORDS: Optical impression, intra oral scanner, bench top scanner, conventional impression, in silico super imposition.

\footnotetext{
* Assistant Professor, Department of Prosthodontics, Faculty of Dentistry, Alexandria University, EGYPT.
} 


\section{INTRODUCTION}

Digital or optical impressions, made with intra oral scanners (IOS), are becoming of an increased use due to their accuracy, ease of use compared to conventional impressions, and comfort to both operators and patients. ${ }^{1,2}$ Several studies have reported that the scanning techniques of the edentulous jaws could capture the details of the edentulous mandibular and maxillary arches, with no significant differences to conventional impressions, for the production of complete dentures with no need for conventional records transferred to the dental laboratory. ${ }^{3-6}$ Other studies further reported that complete dentures fabricated with digital techniques had more retention and stability compared to those produced by conventional techniques, ${ }^{7,8}$ and in spite the fact that optical impressions are considered mucostatic, ${ }^{6}$ another study found that their use to produce computer aided design/computer aided manufacturing (CAD-CAM) denture bases had enough accuracy to preclude the need of functional conventional impressions. ${ }^{9}$

However, other studies concluded that the available IOS technology was not able to trace mobile and smooth tissues of the edentulous arches, especially at the peripheral borders and soft palate, ${ }^{10}$, 11 which affected the trueness of the scanning process, ${ }^{12}$ and rendered such technique only suitable as a replacement of preliminary impressions when other accurate conventional impression techniques were kept in mind. ${ }^{11,13}$ Other controversies about the use of digital scanning procedure versus conventional impression techniques included the time consumed, which still have no significant difference, ${ }^{14}$ the ability to produce better details, ${ }^{15}$ which is doubted when compared to the conventional impression techniques that selectively apply pressure to the edentulous ridges and functionally mold the peripheral limiting structures, ${ }^{16}$ and the clinical reliability of the used IOS. ${ }^{17-19}$

This study compared the optical impressions to the conventional impressions of the edentulous maxillary and mandibular arches with a digital superimposition process, and calculated the values of their deviations from each other.

\section{MATERIALS AND METHODS}

The aim of the current study was to compare two different impression techniques, the optical and the conventional, via computer guided (in silico) super imposition process, and find out if optical impressions, generated with one of the currently available intra oral scanning technologies, specifically the tri-angulation that uses white light to capture images and record videos to create the three dimensional models, can be considered a viable replacement of conventional impressions utilized to make complete dentures.

Eighteen completely edentulous male patients participated in this study. The patients signed an informed consent after explaining the procedures to them, the patients age ranged from 60 to 65 years old. Both conventional impressions and optical impressions were made by the same prosthodontist, and were digitally super imposed and analyzed for surface topographic mismatching.

Patients inclusion criteria included: patients requiring upper and lower complete dentures with healthy oral mucosa, and free of COVID-19. Patients exclusion criteria included: recent extractions, abused mucosa due to old ill-fitting dentures, flappy mucosa and residual ridges having severe bony or soft tissue undercuts that require pre-prosthetic rehabilitation, diabetes mellitus, cardiovascular diseases, and patients who tested positive for COVID-19.

At the first visit, preliminary impressions were made using irreversible hydrocolloid (BMS DENTAL, Italy), and the patients were instructed not to use their old dentures for at least 48 hours before the second visit. The preliminary impressions were poured into dental plaster (Regular set lab plaster, TALLADIUM, USA) to produce the study 
models upon which individual custom impression trays were fabricated using self-cured acrylic resin (Special Tray, DeguDent GmbH, Germany). The individual trays had 4 tissue stops, relieved at hard and sensitive structures, and were closely fitting to the crest of the upper and lower edentulous ridges, maxillary tuberosities, and mandibular retromolar pads and buccal shelves of bone.

At the second visit, optical impressions were made before the conventional definitive impressions. The optical impressions were made using the CEREC Omnicam (Sirona dental Systems GmbH, Germany), for convenience of the right handed operator, the scanning procedure started at the left maxillary tuberosity in a zigzag motion from the peripheral limiting structures of the buccal vestibule to the palatal side of the edentulous ridge, proceeding to the right tuberosity, and finally scanning the rest of the hard palate and the soft palate posterior palatal seal area. Scanning of the mandibular edentulous arches followed the same pattern starting at the left retro molar pad, proceeding to the right retro molar pad while scanning the residual ridge, buccal and lingual vestibules. During such procedure, the cheeks, lips, and tongue were retracted properly. The captured optical details were saved for each patient as a Standard Tessellation Language (STL) file, and the borders of the virtual impressions were trimmed properly using the Sirona software scissors as seen in figures 1 and 2 .

Following the making of the optical impressions, the conventional final impressions were made by peripheral molding of the individual trays using green compound sticks (manufactured by Spofa Dental, Czech Republic, for Kerr corporation, USA) with working temperature $50-51^{\circ} \mathrm{C}$, and Zinc-Oxide eugenol impression material (Cavex Outline, Cavex Holland BV), these impressions were poured into type III hard dental stone (Model Hard Stone, ENRST HIRNICHS Dental GmbH, Germany), and after setting, the produced master casts were scanned using Kavo bench top scanner (Kavo ARCTICA AutoScan), and the scans were saved for their corresponding patients as a Standard Tessellation Language (STL) file as seen in figures 3 and 4 .

The three dimensional superimposition process was carried out using the Geomagic software (Geomagic Qualify 2013, Geomagic, Morrisville, NC, USA), the base and the land areas of the virtual master casts scans were trimmed for the sake of comparison with the clinically captured intra oral scans, the best-fit feature of the software was used to superimpose the clinical and the master cast scans of each patient using the labial and buccal frena, and hamular notches as assembly points, then the $3 \mathrm{D}$ compare feature of the software was used to detect the vertical deviations of the scans from each other. The detected deviations were presented as surface color maps, with each color representing a $0.1 \mathrm{~mm}$ positive or negative deviation.

In each maxillary arch, almost equally spaced 12 readings were taken from the anatomical regions of the buccal vestibule, residual ridge, median palatine raphe and soft palate, and double this number, 24 readings, were taken from the hard palate due to its larger surface area. Also, in each mandibular arch, 12 readings were taken from each anatomical regions of the buccal vestibule, lingual vestibule, residual ridge, and 6 readings for each right and left retromolar pads, and right and left buccal shelves of bone as seen in figures 1 and 2. The Geomagic software "tabular view-3D compare" was used to provide the value for each of these super imposition readings. The average reading from each anatomical region of each arch was calculated, then the readings from the 18 patients were tabulated for statistical analysis using the Kruskal-Wallis test of the Statistics Package for the Social Sciences Software (SPSS version 23.0, SPSS Inc., Chicago, IL, USA) to compare the impressions surface matching deviations, with $P<.05$ indicating statistical significance. 


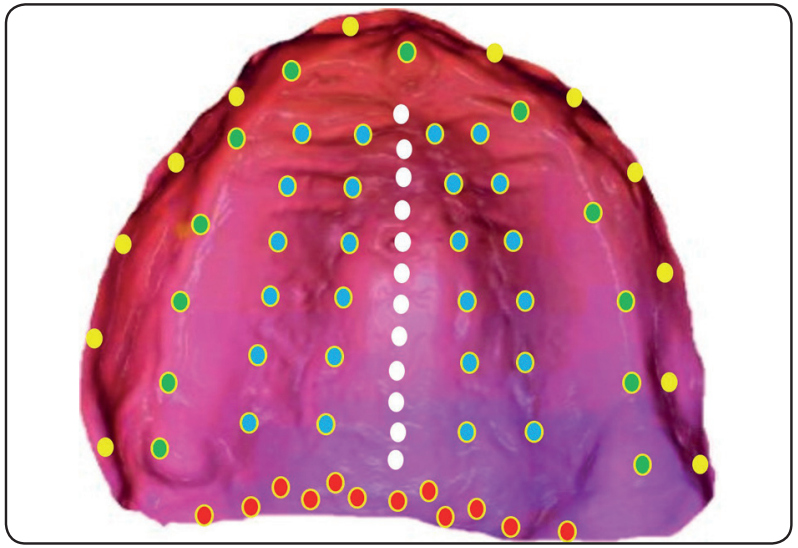

Fig. (1) Intra oral scan of the maxillary arch with approximate locations of the vertical deviation readings; the yellow dots refer to the buccal vestibule, the green dots refer to the residual ridge, the light blue dots refer to the hard palate, the white dots refer to the median palatine raphe, and the red dots refer to the soft palate.

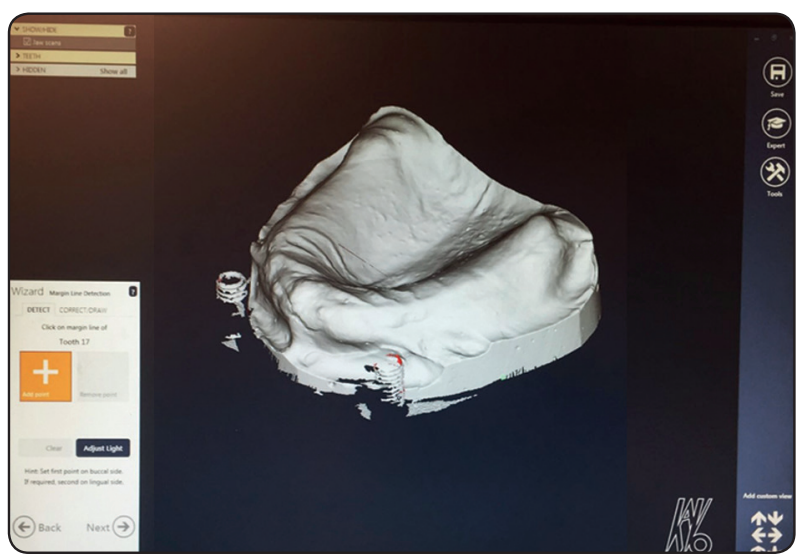

Fig. (3) Laboratory scan of the maxillary master cast

\section{RESULTS}

Figure 5 shows a color map of one of the studied maxillary arches, and figure 6 shows the distribution of the optical impressions variation values from the conventional impressions, where the mean and standard deviation for the buccal vestibule region were $0.84+0.07$, for the residual ridges were 0.12 +0.01 , for the hard palate were $0.17+0.02$, for the median palatine raphe were $0.19+0.03$, and for the soft palate were $0.24+0.02$ as seen in table 1. The only statistically significant difference in the deviation of the optical from the conventional

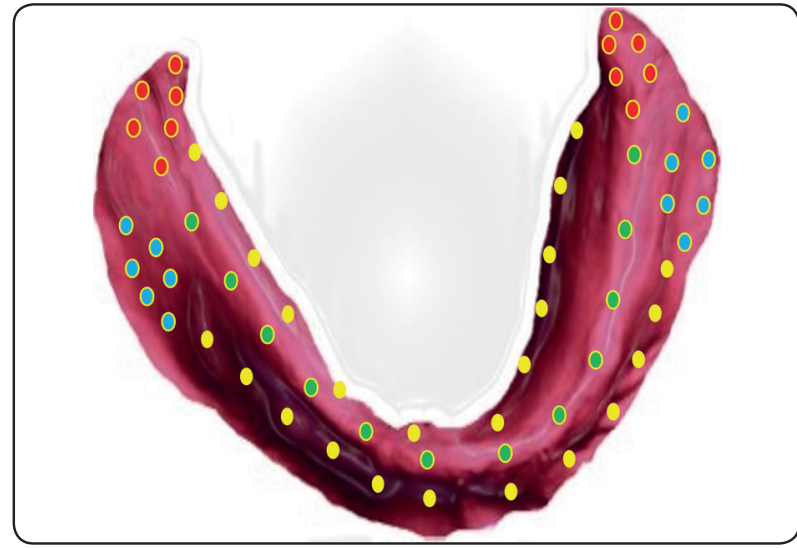

Fig. (2) Intra oral scan of the mandibular arch with the approximate locations of the vertical deviation readings; the yellow dots refer to the buccal and lingual vestibules, the light blue dots refer to the buccal shelves of bone, the green dots refer to the residual ridge, and the red dots refer to the retromolar pad.

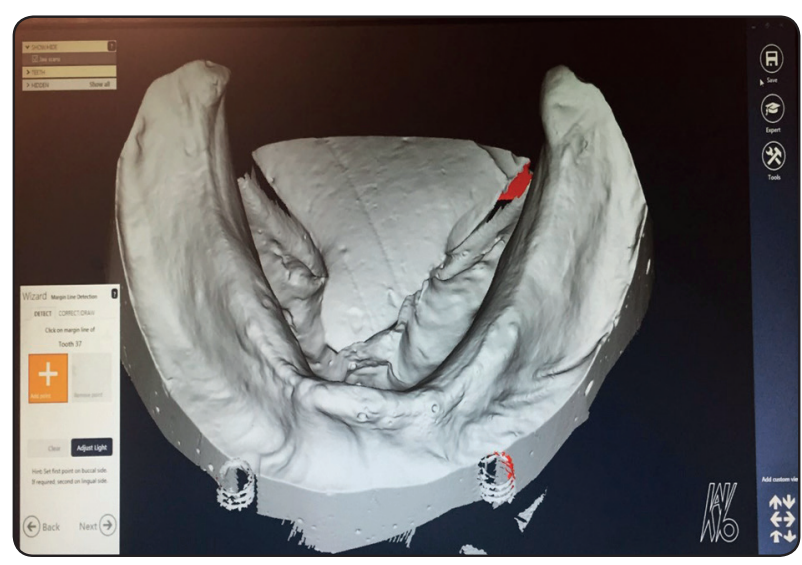

Fig. (4) Laboratory scan of the mandibular master cast

impressions was found in the region of the buccal vestibules as proven by the value of $p( \pm 0.01)$ in table 1 , meaning that optical impressions were able to register all the maxillary arches surface details as the conventional impressions except at the depth of the buccal vestibules.

Figure 7 shows a color map of one of the studied mandibular arches, and figure 8 shows the distribution of the optical impressions variation values from the conventional impressions, where the mean and standard deviation for the buccal vestibules regions were $0.95+0.02$, for the retromolar pad $0.80+0.06$, 
for the residual ridge $-0.01+0.06$, for the buccal shelves of bone $0.82+0.08$, and for the lingual vestibules $0.91+0.05$ as seen in table 2 . There were statistically significant differences in the deviation of the optical from the conventional impressions in all the mandibular arches anatomical regions except

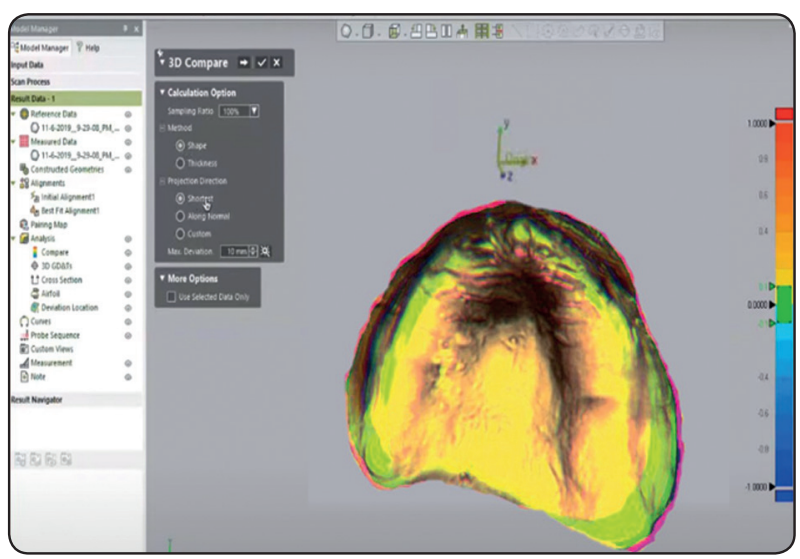

Fig. (5) Super imposition of the maxillary intra oral scan and maxillary master cast scan: green areas represented minimal displacements ranging from -0.1 to $+0.1 \mathrm{~mm}$, red areas represented $+1 \mathrm{~mm}$ as maximum positive deviations, and blue areas represented $-1 \mathrm{~mm}$ as maximum negative deviations. for the residual ridges as proven by the value of $p(=0.1)$ in table 2 , meaning that optical impressions were not able to register any of the maxillary arches surface details as the conventional impressions except at the residual ridges.

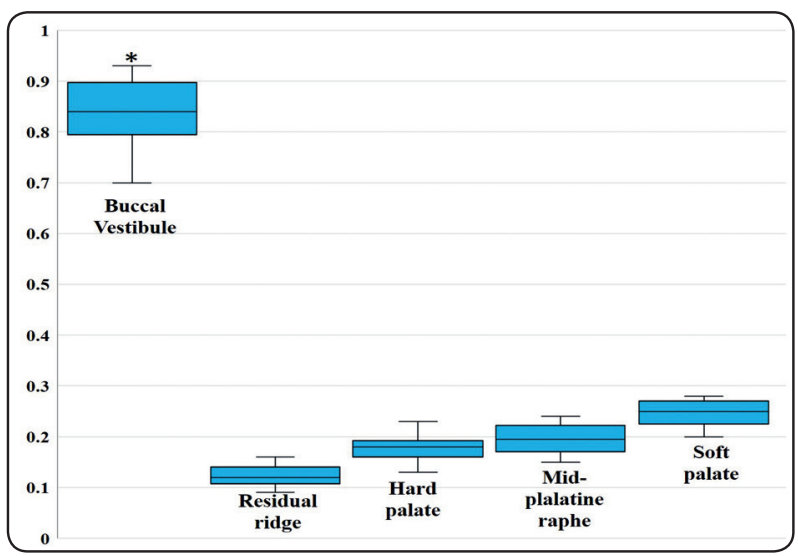

Fig. (6) Box plot chart of optical impressions variations from conventional impressions of the maxillary arches, the rectangle boxes show the different anatomical regions of the maxillary arches, the horizontal lines in the boxes show the median, the whiskers above and below the rectangles show the maximum and minimum values, and the ${ }^{(*)}$ denotes statistical significance.

TABLE (1) Maxillary arches optical impressions vertical deviation values from conventional impressions

\begin{tabular}{|c|c|c|c|c|c|}
\hline & Buccal Vestibule & Residual ridge & Hard palate & Mid-palatine raphe & Soft palate \\
\hline Mean + standard deviation & $0.84+0.07$ & $0.12+0.01$ & $0.17+0.02$ & $0.19+0.03$ & $0.24+0.02$ \\
\hline Kruskal Wallis $p$ & 0.01 & 0.06 & 0.06 & 0.07 & 0.09 \\
\hline
\end{tabular}

TABLE (2) Mandibular arches optical impressions vertical deviation values from conventional impressions.

\begin{tabular}{|c|c|c|c|c|c|}
\hline & Buccal Vestibule & Retromolar pad & Residual ridge & Buccal shelf & Lingual vestibule \\
\hline Mean + standard deviation & $0.95+0.02$ & $0.80+0.06$ & $-0.01+0.06$ & $0.82+0.08$ & $0.91+0.05$ \\
\hline Kruskal Wallis $p$ & 0.01 & 0.02 & 0.1 & 0.03 & 0.01 \\
\hline
\end{tabular}




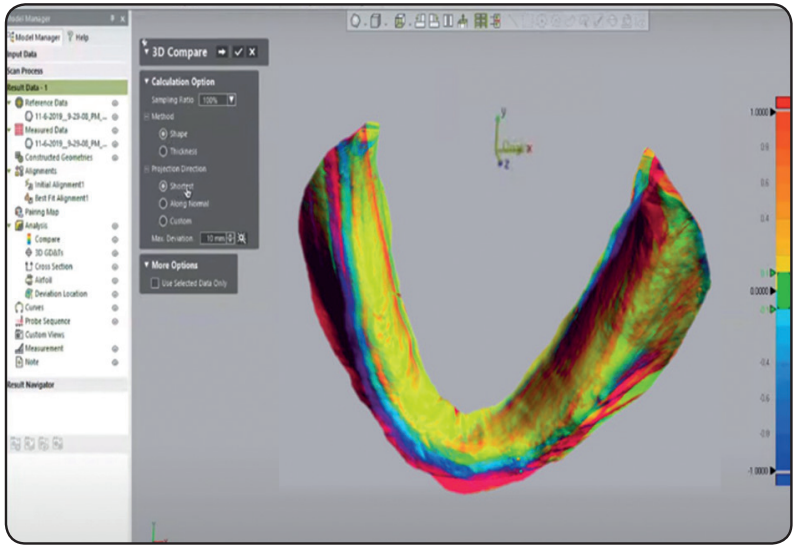

Fig. (7) Super imposition of the mandibular intra oral scan and mandibular master cast scan: green areas represented minimal displacements ranging from -0.1 to $+0.1 \mathrm{~mm}$, red areas represented $+1 \mathrm{~mm}$ as maximum positive deviations, and blue areas represented $-1 \mathrm{~mm}$ as maximum negative deviations

\section{DISCUSSION}

This study compared the deviation of optical impressions from the conventional impressions using an in silico approach, with controlled selection of the study participants, as no edentulous ridges with redundant, flappy tissues, or undercuts were included. According to Chandran et $\mathrm{al}^{1}$ and Priyanka et $\mathrm{al}^{2}$, the use of IOS is becoming more popular in capturing the details of digital impressions with operator and patients' preference, as they are more comfortable, reduce the number of visits, and have superior accuracy to conventional impressions. Fang et $\mathrm{al}^{3,8}$ added that these scanners were able to capture the morphology of edentulous arches, and helped the full digital production of complete dentures, with improved retention and stability over those produced by conventional techniques. Goodacre et $\mathrm{al}^{6}$ described a successful technique to digitally fabricate complete dentures using IOS, and acknowledged that optical impressions were truly mucostatic, Srinivasan et $\mathrm{al}^{9}$ similarly described a digital technique, and suggested that $\mathrm{CAD} / \mathrm{CAM}$ dentures may not require functional impressions due to their precise manufacturing, and lack of

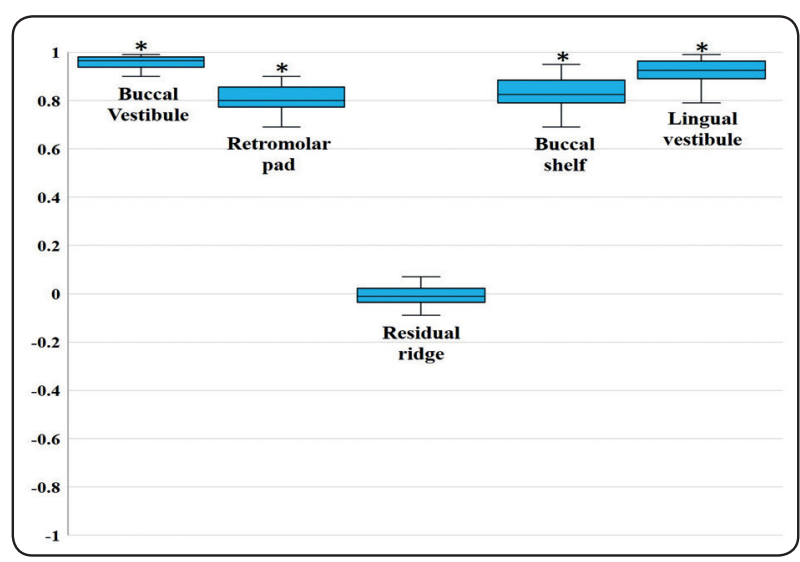

Fig. (8) Box plot chart of optical impressions variations from conventional impressions of the mandibular arches, the rectangle boxes show the different anatomical regions of the maxillary arches, the horizontal lines in the boxes show the median, the whiskers above and below the rectangles show the maximum and minimum values, and the $(*)$ denotes statistical significance.

distortion of the denture bearing tissues during the optical impression procedure, Lo Russo and Salamini $^{22}$ claimed that in such situation, denture retention depends on the surface tension of the saliva between the denture and the oral mucosa, and proper peripheral seal may not be required, and Regis et $\mathrm{al}^{27}$ reported that the making of two impression, preliminary and definitive, was not found to be essential for clinical success of complete dentures and better masticatory function. All of these findings were further supported by Jung et $\mathrm{al}^{4}$ who reported that there was no statistically significant difference between the optical and conventional impressions of completely edentulous patients, and Al Helal et $\mathrm{al}^{7}$ who concluded that the retention of pre-polymerized CAD/CAM milled complete dentures was higher than that of the conventionally fabricated heat polymerized complete dentures.

However, it could be true that peripheral seal may not be needed for implant assisted prostheses as suggested by Hack et $\mathrm{al}^{10}$, but proper extension of the denture margins, that were adjusted with green compound peripheral molding and a layer of zincoxide eugenol, was found to be essential and crucial 
for the complete dentures peripheral valve seal and retention, especially for the lower dentures, as confirmed by the findings of Jung et $\mathrm{a}^{14}$, D'Arienzo et all $^{11}$, Tasaka et $\mathrm{al}^{12}$, Zarone et $\mathrm{al}^{13}$, Ender et $\mathrm{al}^{23}$, and Chebib et $\mathrm{al}^{26}$, these findings rendered current optical impressions not suitable as a replacement of complete dentures conventional impressions, these findings also came in agreement with the results of this study, which showed that in the maxillary arches there was no statistically significant difference between the optical and conventional impressions except at the depth of sulcus of the buccal vestibules, and in the mandibular arches there were statistically significant differences in the deviation of the optical from the conventional impressions in the depth of sulcus of the buccal and lingual vestibules, buccal shelves of bone, and retromolar pad areas. The results of this study also came in agreement with Hack et $\mathrm{al}^{10}$, and D'Arienzo et $\mathrm{al}^{11}$ and confirmed the inability of the IOS, used in this study, to trace smooth structures in the previously mentioned anatomic region and exert similar pressure applied by the conventional impressions. However, in this study there was no statistically significant difference recorded in the soft palate regions, where the optical impressions mean deviation from the conventional impressions was $0.24 \mathrm{~mm}$, a value which was thought to be within the limits of accepted range of clinical accuracy for the impressions to be accepted for the making of complete dentures, that is $0.5 \mathrm{~mm}$, as determined by Hack et al. ${ }^{10}$

In contrast to the Lava Chairside Oral scanner used by Hack et al, ${ }^{10}$ the Omnicam IOS used in this study did not need the use of a powder, which could affect the scanning process by its thickness or unequal distribution. The Omnicam was also found to properly capture soft tissue details when used in a zigzag motion as advocated by Lo Russo and Salamini, ${ }^{22}$ although Medina-Sotomayor et al ${ }^{17}$, ${ }^{18}$ found that the Omnicam trueness and precision were not affected by any certain scanning strategy, and that it had a positive relationship between its resolution and precession as compared to True Definition, Trios, and iTero scanners. In other words, this meant that only one scan by Omnicam was enough to capture the details of the edentulous arches compared to the 3 scans needed in the work of Hack et $\mathrm{al}^{10}$ as the Omnicam scans showed a high degree of trueness in terms of measurements closeness to actual values and precision in terms of consistency in repeated measures, yet, it remained a non-contact scanner that did not selectively displace the tissues as conventional impressions.

The limitations of this study included the followings: first, only one type of IOS was used, however, there are no currently available IOS that could add to the findings of this study since they all are non-contact scanners; second, the intra oral scans were compared to the scans of a bench top scanner, which is more accurate, and worked in absence of the oral limiting conditions such as saliva and tissue resilience, however, bench top scanners bulk renders it impossible for use in the oral cavity; third, the master casts scans, which were used as the gold standard to which the intra oral scans were compared, suffered the dimensional changes of the impression material and dental cast stone material, however, the mean dimensional changes detected in this study for optical impressions were much greater than the impression material and stone dimensional changes.

The previously mentioned limitations together with the recommendation of Mangano et al, ${ }^{19,28}$ that intra oral scanners are not yet suitable for registering the details of edentulous arches, render the available IOS technologies no useful, however, several other authorities ${ }^{29-32}$ have encouraged the full digital transformation of removable prosthetics work flow due to the undeniable benefits of the CAD/CAM milled prostheses, which use better materials, with better mechanical properties, fit, and overcome the shortcomings of the conventional techniques, such as denture base distortion due to porosity or polymerization shrinkage. 
In conclusion, the results of this study showed that optical impressions were not able to replace conventional impressions for completely edentulous arches, yet, the promising advantages of full digital work flows call for the development of specifically designed soft tissue scanners, with appropriate head size and design, suitable for the functional depth of peripheral sulci, ${ }^{13}$ with enough pixel and voxel recording to register mobile and soft tissues, and software that enable the placement of extra pressure or relieve on the soft tissues at strategic anatomical locations in the edentulous arches.

\section{CONCLUSION}

Within the limitations of this study, it was concluded that optical impressions were not able to register the functional depth of sulci in the maxillary and mandibular edentulous arches, neither the selectively pressed buccal shelves of bone and retromolar pads in the mandibular edentulous arches. However, due to the fact that digital transformation in prosthodontics is inevitable, further research is required to improve the quality of intra oral scanning of edentulous arches.

\section{REFERENCES}

1. Chandran SK, Jaini J, Babu AS, Mathew A, Keepanasseril A. Digital Versus Conventional Impressions in Dentistry: A Systematic Review. Journal of Clinical \& Diagnostic Research. 2019 Apr 1;13(4).

2. Priyanka G, Sujesh M, Kumar R, Rao C, Srujana Z. Digital impressions in prosthodontics-past, present and future trends. IP Annals of Prosthodontics and Restorative Dentistry. 2020;6(2):66-70.

3. Fang Y, Fang JH, Jeong SM, Choi BH. A technique for digital impression and bite registration for a single edentulous arch. Journal of Prosthodontics. 2019 Feb; 28(2): e519-23.

4. Jung S, Park C, Yang HS, Lim HP, Yun KD, Ying Z, Park SW. Comparison of different impression techniques for edentulous jaws using three-dimensional analysis. The Journal of Advanced Prosthodontics. 2019 Jun 1;11(3):179-86.
5. Fang JH, An X, Jeong SM, Choi BH. Digital intraoral scanning technique for edentulous jaws. The Journal of prosthetic dentistry. 2018 May 1;119(5):733-5.

6. Goodacre BJ, Goodacre CJ, Baba NZ. Using Intraoral Scanning to Capture Complete Denture Impressions, Tooth Positions, and Centric Relation Records. International Journal of Prosthodontics. 2018 Jul 1;31(4).

7. AlHelal A, AlRumaih HS, Kattadiyil MT, Baba NZ, Goodacre CJ. Comparison of retention between maxillary milled and conventional denture bases: a clinical study. The Journal of prosthetic dentistry. 2017 Feb 1;117(2):233-8.

8. Fang JH, An X, Jeong SM, Choi BH. Digital immediate denture: A clinical report. The Journal of prosthetic dentistry. 2018 May 1;119(5):698-701.

9. Srinivasan M, Kalberer N, Naharro M, Marchand L, Lee H, Müller F. CAD-CAM milled dentures: The Geneva protocols for digital dentures. The Journal of Prosthetic Dentistry. 2020 Jan 1;123(1):27-37.

10. Hack G, Liberman L, Vach K, Tchorz JP, Kohal RJ, Patzelt SB. Computerized optical impression making of edentulous jaws-An in vivo feasibility study. Journal of Prosthodontic Research. 2020 Feb 13.

11. D'Arienzo LF, D’Arienzo A, Borracchini A. Comparison of the suitability of intra-oral scanning with conventional impression of edentulous maxilla in vivo. A preliminary study. Journal of Osseointegration. 2018 Nov $14 ; 10(4): 115-20$.

12. Tasaka A, Uekubo Y, Mitsui T, Kasahara T, Takanashi T, Homma S, Matsunaga S, Abe S, Yoshinari M, Yajima Y, Sakurai K. Applying intraoral scanner to residual ridge in edentulous regions: in vitro evaluation of inter-operator validity to confirm trueness. BMC oral health. 2019 Dec; 19(1):1-0.

13. Zarone F, Ruggiero G, Di Mauro MI, Spagnuolo G, Ferrari M, Sorrentino R. Accuracy of Three Impression Materials on the Totally Edentulous Maxilla: In Vitro/In Silico Comparative Analysis. Materials. 2020 Jan;13(3):515.

14. Cicciù M, Fiorillo L, D'Amico C, Gambino D, Amantia EM, Laino L, Crimi S, Campagna P, Bianchi A, Herford AS, Cervino G. 3D Digital Impression Systems Compared with Traditional Techniques in Dentistry: A Recent Data Systematic Review. Materials. 2020 Jan;13(8):1982.

15. Bonnet G, Batisse C, Bessadet M, Nicolas E, Veyrune JL. A new digital denture procedure: a first practitioners appraisal. BMC oral Health. 2017 Dec 1;17(1):155. 
16. Malachias A, Paranhos HD, Silva CH, Muglia VA, Moreto C. Modified functional impression technique for complete dentures. Brazilian dental journal. 2005 Aug;16(2):135-9.

17. Medina-Sotomayor P, Pascual-Moscardó A, Camps I. Relationship between resolution and accuracy of four intraoral scanners in complete-arch impressions. Journal of clinical and experimental dentistry. 2018 Apr; 10(4):e361.

18. Medina-Sotomayor P, Pascual M A, Camps A I. Accuracy of four digital scanners according to scanning strategy in complete-arch impressions. PloS one. 2018 Sep 13;13(9):e0202916.

19. Mangano FG, Admakin O, Bonacina M, Lerner H, Rutkunas V, Mangano C. Trueness of 12 intraoral scanners in the full-arch implant impression: a comparative in vitro study. BMC Oral Health. 2020 Dec;20(1):1-21.

20. Rhee YK, Huh YH, Cho LR, Park CJ. Comparison of intraoral scanning and conventional impression techniques using 3-dimensional superimposition. The journal of advanced prosthodontics. 2015 Dec 1;7(6):460-7.

21. Patzelt SB, Vonau S, Stampf S, Att W . Assessing the feasibility and accuracy of digitizing edentulous jaws. J Am Dent Assoc 2013;144(8):914-20.

22. Lo Russo L, Salamini A . Single-arch digital removable complete denture: a workflow that starts from the intraoral scan. J Prosthet Dent 2017;120(1):20-4.

23. Ender A, Mehl A. Influence of scanning strategies on the accuracy of digital intraoral scanning systems. Internat $\mathbf{J}$ Computerized Dentistry 2013; 16:11- 21.

24. Kim JE, Amelya A, Shin Y, Shim JS. Accuracy of intraoral digital impressions using an artificial landmark. J Prosthet Dent. 2017;117:755-61.
25. Fang JH, An X, Jeong SM, Choi BH. Development of complete dentures based on digital intraoral impressionscase report. J Prosthodont Res. 2018; 62:116-20.

26. Chebib, N.; Kalberer, N.; Srinivasan, M.; Maniewicz, S.; Perneger, T.; Müller, F. Edentulous jaw impression techniques: An in vivo comparison of trueness. J. Prosthet. Dent. 2019, 121, 623-630.

27. Regis, R.R.; Alves, C.C.; Rocha, S.S.; Negreiros, W.A.; Freitas-Pontes, K.M. The importance of a two-step impression procedure for complete denture fabrication: A systematic review of the literature. J. Oral Rehabil. 2016, $43,771-777$.

28. Mangano, F.; Gandolfi, A.; Luongo, G.; Logozzo, S. Intraoral scanners in dentistry: A review of the current literature. BMC Oral Health 2017, 17, 149.

29. Bilgin, M.S.; Baytaro glu, E.N.; Erdem, A.; Dilber, E. A review of computer-aided design/computer-aided manufacture techniques for removable denture fabrication. Eur. J. Dent. 2016, 10, 286-291.

30. AlHelal, A.; AlRumaih, H.S.; Kattadiyil, M.T.; Baba, N.Z.; Goodacre, C.J.Comparison of retention between maxillary milled and conventional denture bases: A clinical study. J. Prosthet. Dent. 2017, 117, 233-238.

31. McLaughlin, J.B.; Ramos, V., Jr.; Dickinson, D.P. Comparison of Fit of Dentures Fabricated by Traditional Techniques Versus CAD/CAM Technology. J. Prosthodont. 2019, 28, 428-435.

32. Bidra, A.S.; Taylor, T.D.; Agar, J.R. Computer-aided technology for fabricating complete dentures: Systematic review of historical background, current status, and future perspectives. J. Prosthet. Dent. 2013, 109, 361-366. 